\title{
Reverse posterior diaphragm plication for the treatment of intraoperative voluminous chylothorax
}

\author{
Leonardo Duranti, Alessandro Pardolesi, Luca Tavecchio, Luigi Rolli, Ugo Pastorino \\ Thoracic Surgery Unit, IRCCS Istituto Nazionale dei Tumori Foundation, Milan, Italy \\ Correspondence to: Leonardo Duranti, MD, PhD. Via Venezian1 20133 Milan, Italy. Email: leonardo.duranti@istitutotumori.mi.it.
}

\begin{abstract}
Chylothorax is an uncommon but severe complication, often challenging to treat. In this study, we describe an alternative surgical technique for the management of intraoperative chylothorax occurred in young male patients that underwent left thoracotomy for resection of metastatic teratoma.
\end{abstract}

Keywords: Diaphragm; chylothorax

Received: 04 September 2018; Accepted: 26 November 2018; Published: 06 December 2018.

doi: $10.21037 /$ shc.2018.11.07

View this article at: http://dx.doi.org/10.21037/shc.2018.11.07

\section{Introduction}

Chylothorax is a relatively rare, but potentially lifethreatening complication. Iatrogenic injury of the thoracic duct frequently causes postoperative chylothorax, more frequently performing extended dissection of the posterior mediastinum (1). Although conservative treatment including chest total parental nutrition and chest drainage is frequently advocated as initial treatment, the surgical approach is often required due to the failure of such therapy, and it can be represented by thoracic duct closure, or in selected case, by pleurectomy and eventual pleurodesis (2). In this study, we report a surgical technique adopted for the management of intraoperative voluminous chylus effusion in a young adult patient who underwent sequential bilateral thoracotomy for metastatic mature teratoma.

\section{Case presentation}

A 38 years old man was referred to our Division for the treatment of metastatic mature teratoma. Patient past medical history began in 2008 when he underwent neoadjuvant chemotherapy (bleomycin, etoposide, cisplatin for four cycles) followed by left orchifuniculectomy and bilateral retroperitoneal lymphadenectomy for the treatment of mature teratoma. One year later in 2009, a mediastinal relapse was diagnosed, and the patient underwent retrocrural and mediastinal lymphadenectomy through a right lateral thoracotomy. Removal of all the mediastinal lesions required an extended dissection of the posterior mediastinum and due to intra-operative lymphatic leak the thoracic duct was then isolated and sutured. However, in the postoperative time the patient developed massive chylothorax requiring a right redo-thoracotomy with chemical talc pleurodesis. The postoperative course was uneventful.

In 2018 for a new metastatic retrocrural adenopathy we performed a left lateral muscle sparing thoracotomy. Due to previous contralateral dissection on the posterior mediastinal fat tissue around the descending aorta and the oesophagus we had to perform a full $5 \mathrm{~cm}$ left phrenotomy to identify the adenopathy. Dissection was complicated by distal retroperitoneal bleeding that required a 3 minutes clamping of the aorta to perform a suture safely, and we were able to stop the bleeding. We then isolated and dissected the adenopathy that was radically removed together with all the surrounding fat tissue, suturing and clipping all the tissue around the aorta. Once lymphadenectomy was completed pleural effusion (around $300 \mathrm{~mL}$ in less than 15 minutes) became visible arising from the retroperitoneum into the left chest cavity. We performed intra-operative biochemical test that confirmed the initial suspect of chylothorax (pleural fluid triglyceride level of $138 \mathrm{mg} / \mathrm{dL}$ ). We sutured the mediastinal pleural and the phrenotomy, but the chylous effusion was still voluminous, so we considered an alternative technique, As the thoracic 

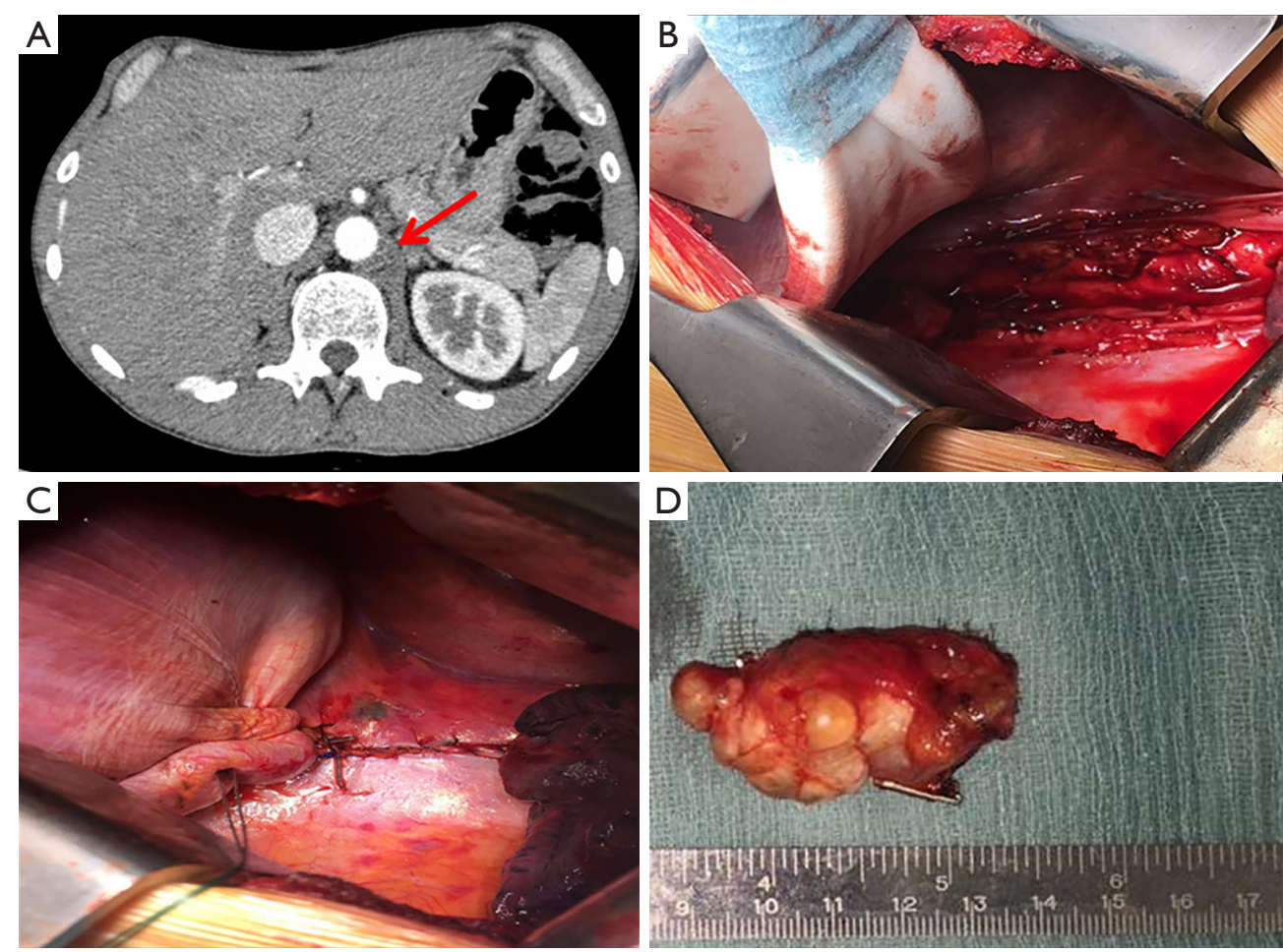

Figure 1 A 38 year-old man with a mediastinal teratoma recurrence. (A) CT scan with a red arrow on the residual mature teratoma; (B) left aortic subadventitial dissection. Surgical retractor on the diaphragm; (C) the reverse flap of diaphragm anchored to the chest wall and the pleural flap covering the descending thoracic aorta; (D) 3-cm Teratoma.

duct have been ligated during previous aright surgical procedure. We performed a diaphragmatic plication fixed posteriorly on the chest wall so to interrupt communication between retroperitoneum and left chest cavity. We created a muscular pocket—patch posteriorly overturned and fixed in 3 steps: (I) two ring forceps elevated the redundant diaphragm and after accurate palpation to confirm the absence of abdominal viscera a Satinsky clamp was placed so to create a fold and two monofilaments polyglyconate absorbable U stitches (Maxon MT20, 48 mm 1/2-blunt taper needle) were passed at the base of the fold; (II) these two stitches were then fixed to the posterior diaphragm; (III) we finally passed other two stitches between the tip of the fold (Maxon MT20, $48 \mathrm{~mm} \mathrm{1/2-blunt} \mathrm{taper} \mathrm{needle)} \mathrm{and} \mathrm{the} \mathrm{chest}$ wall, precisely around the posterolateral portion of the 11th rib, pulling-up the plicated diaphragm against the costal arch. Once the procedure was completed, the leak was no more evident (Figure 1). At the end of the procedure, we placed a 24-French chest drain.

We preferred to adopt an alipidic oral diet for the seven days and then we performed the «milk stress test» with no sign of chylothorax. So, we removed the chest drain and the patient was discharged after in 10th postoperative day. After six months of follow-up chest and abdominal CT scan did not show any signs of peritoneal or pleural effusion. The patient is alive with no evidence of disease.

\section{Discussion}

The medical therapy with octreotide (3) should be considered in patients not considered feet for redo-surgery or in case re-operation is not successful. The Denver shunt is permanent and uncomfortable, but in case of unsuccessful conservative treatment, in non-surgical patients, represents a valid alternative treatment; however, in young/adult patients with good oncological prognosis, it should be considered only in case of failure of other treatments (4).

Our patient had a poor respiratory function, and due to previous right talc pleurodesis, a left pleurectomy could have increased the risk of postoperative respiratory complication. This type of plication is different from the others employed for phrenic nerve paralysis because it is unnecessary to get a high tension on the sutured diaphragm, the diaphragmatic muscle is tonic, and there will be no loss 
in respiratory function.

Although experienced just in one case, our posterior diaphragmatic plication should be considered for chylous leakage that origins from unidentified retroperitoneal area as a patch separating the abdomen from the pleura and preventing the pump pleural mechanism (5) in difficult situations, such as in this case or in contralateral postpneumonectomy chylothorax after great mediastinal surgery.

\section{Acknowledgements}

Thanks to Priscilla Errigo and Lorenzo Fenini for informatic support.

\section{Footnote}

Conflicts of Interest: The authors have no conflicts of interest to declare.

Informed Consent: Written informed consent was obtained from the patient for publication of this manuscript and any accompanying images.

\section{References}

1. Cerfolio R, Allen M, Deschamps C, et al. Postoperative chylothorax. J Thorac Cardiovasc Surg 1996;112:1361-5.

2. Martucci N, Tracey M, Rocco G. Postoperative chylothorax. Thorac Surg Clin 2015;25:523-8.

3. Markham KM, Glover JL, Welsh RJ, et al. Octreotide in the treatment of thoracic duct injuries. Am Surg 2000;66:1165-7.

4. Hanlon R, Lee J, Fenton-Lee D, et al. Pleuroperitoneal Denver shunt insertion for the treatment of refractory chylothorax in a patient with tuberous sclerosis complex and lymphangioleiomyomatosis. Intern Med J 2017;47:1463-4.

5. Leo F, Girotti P, Tavecchio L, et al. Anterior diaphragmatic plication in mediastinal surgery: the "reefing the mainsail" technique. Ann Thorac Surg 2010;90:2065-7.

doi: $10.21037 /$ shc.2018.11.07

Cite this article as: Duranti L, Pardolesi A, Tavecchio L, Rolli L, Pastorino U. Reverse posterior diaphragm plication for the treatment of intraoperative voluminous chylothorax. Shanghai Chest 2018;2:91. 